\title{
Nanofibers for Regenerative Dentistry: From Scaffolds to Drug Delivery Systems
}

\author{
Marco C. Bottino ${ }^{1}$
}

${ }^{1}$ Department of Biomedical and Applied Sciences (Division of Dental Biomaterials), Indiana University School of Dentistry, Indianapolis, IN, USA.

Periodontitis is one of the most aggressive diseases that impair the integrity of the periodontium. It is a chronic inflammatory disease that affects patients of all ages. The progression of periodontitis leads to the destruction of the periodontium, ultimately leading to tooth loss [1]. The treatment of periodontitis has evolved over the years. Guided tissue and bone regeneration have been increasingly used with the aid of a physical membrane that acts as a barrier to prevent epithelial and connective tissue downgrowth into the defect, enabling periodontal regeneration. Different processing techniques have been employed to fabricate these membranes [1]. Among them, electrospinning, a simple, versatile and welldocumented method has been used to fabricate nano- and micro-scale fibers [1]. The chemical characteristics of the polymer(s) or blends are not compromised during processing, which has allowed investigators to incorporate antimicrobial drugs as well as bioactive nanoparticles (e.g., calcium phosphates and metallic oxides) into different polymers and, therefore, develop a material with therapeutic properties [1]. Furthermore, electrospinning has also allowed the synthesis of tissue specific multilayered scaffolds to simultaneously amplify hard and soft periodontal tissue regeneration.

Meanwhile, traumatic injuries or bacterial infection of the dental pulp lead to inflammation and, if left untreated, to necrosis [2]. In the US, over 15 million patients undergo root canal treatment each year. In children, pulpal necrosis promotes the death of odontoblasts, resulting in root development disruption and often tooth fracture. No regenerative therapy exists that promotes root canal disinfection and can predictably guide the growth and development of pulp and dentin in necrotic teeth. Regrettably, antibiotic pastes used for root canal disinfection prior to a regenerative strategy have been shown to negatively impair dental pulp stem cells (DPSCs) survival [2]. The purpose of this paper is to present an update on the clinical prospects of nanofibers in regenerative dentistry. Here, we highlight the synthesis of bioactive scaffolds for periodontal regeneration and our pioneering work on the development of a cell-friendly disinfection strategy through the fabrication of drug delivery systems based on antibioticcontaining polymer nanofibers for regenerative endodontics.

Poly( $\varepsilon$-caprolactone) (PCL) and PCL/gelatin (PCL/GEL) were dissolved in hexafluoropropanol and loaded with distinct concentrations of zinc oxide $(\mathrm{ZnO})$ nanoparticles. Electrospinning was performed using optimized parameters and the fibers were characterized via scanning and transmission electron microscopies (SEM/TEM), energy dispersive X-ray spectroscopy (EDS), antimicrobial activity against periodontopathogens, and cytotoxicity test using human dental pulp stem cells (hDPSCs). ZnO nanoparticles were successfully incorporated into the overall submicron fibers, which showed fairly good morphology and microstructure. Upon $\mathrm{ZnO}$ nanoparticles' incorporation, the PCL and PCL/GEL fibers became thicker and thinner, respectively. All $\mathrm{ZnO}$-containing membranes displayed antibacterial activity against the bacteria tested, which was generally more pronounced with increased $\mathrm{ZnO}$ content (Fig. 1). All membranes synthesized in this study demonstrated satisfactory cytocompatibility, although the presence of $30 \mathrm{wt} . \% \mathrm{ZnO}$ led to decreased viability (data not shown). Collectively, the data suggest that PCL- and PCL/GEL-based membranes containing a low content of $\mathrm{ZnO}$ nanoparticles can potentially function as a biologically safe antimicrobial membrane for guided tissue/bone regeneration. 
Antimicrobial drugs such as metronidazole, ciprofloxacin, and minocycline were added to a polydioxanone (PDS) polymer solution and spun into nanofibers. Dentin specimens were inoculated with A. naeslundii (ATCC 43146) for 7 days for biofilm formation. The infected dentin specimens were exposed to the triple-antibiotic containing nanofibers to evaluate the antimicrobial efficacy. Confocal laser scanning microscopy was done to determine bacterial viability. Our data demonstrate significant biofilm eradication (Fig. 2), strongly supporting further preclinical evaluation of these novel antibioticcontaining fibers as a disinfection strategy for root canal bacterial biofilm eradication. [3]

\section{References:}

[1] Münchow EA et al, Dent Mater 31 (2015), p. 1038-51.

[2] Albuquerque MT et al, J Dent Res 93 (2014), p. 1222-31.

[3] The author acknowledges the expert TEM assistance of Ms. Caroline Miller (Indiana University).
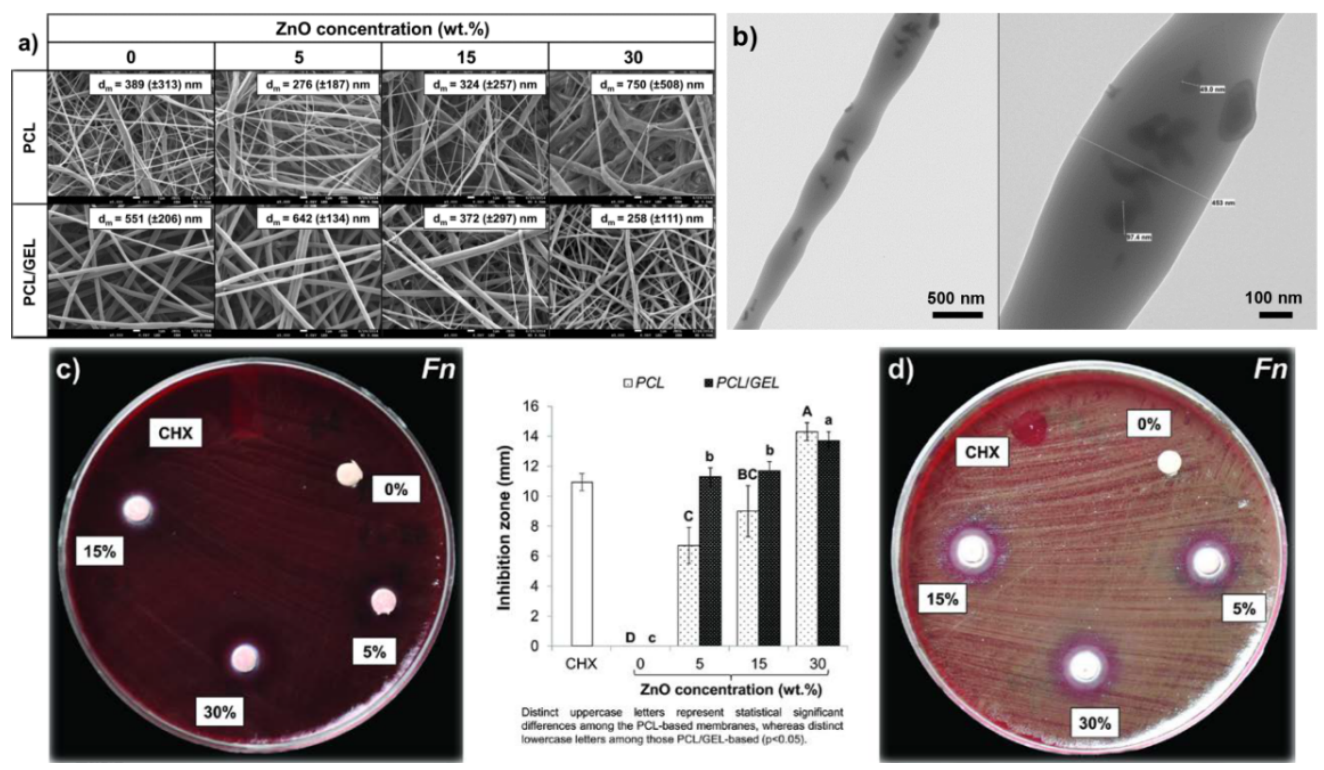

Figure 1. Morphological and antimicrobial analyses of the ZnO-loaded scaffolds (A-B) Scanning and transmission electron microscopy. (B) TEM micrographs showing the presence of $\mathrm{ZnO}$ particles within the fibers. (C-D) Antimicrobial properties against Fusobacterium nucleatum $(F n)$ as a function of the $\mathrm{ZnO}$ content. A $0.12 \%$ chlorhexidine solution ( $\mathrm{CHX}$ ) was used as the positive control [3].
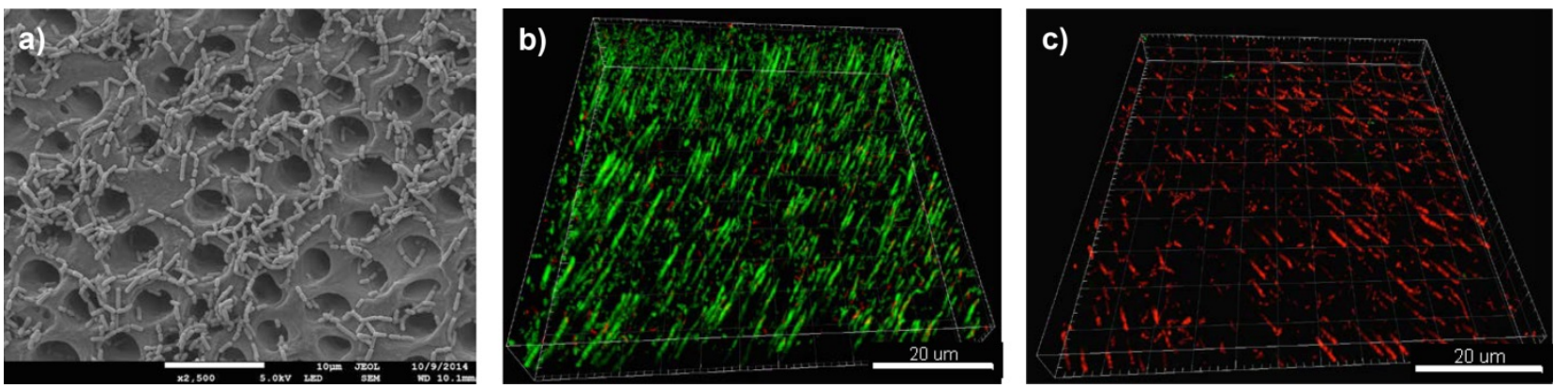

Figure 2. Antimicrobial properties against $\boldsymbol{A}$. naeslundii (An) dentin-infected biofilm. (A) SEM and (B) confocal (green=live bacteria) micrographs of An biofilm on the dentin surface (control). (C) Confocal micrograph showing a significant reduction in the $\%$ of viable bacteria (red=dead bacteria). 